\title{
Research of an Electronic Endoscope Based on ARM
}

\author{
Jian $\mathrm{Li}^{\mathrm{a}}$ and Ningzhou $\mathrm{He}^{\mathrm{b}}$ \\ Information Technology College of Jilin Agricultural University. Changchun 130118, China \\ a342884220@qq.com, b774871296@qq.com
}

Keywords: Electronic endoscope; ARM; Light source; CMOS image sensor; Medical

\begin{abstract}
A flexible medical electronic endoscope with an outside diameter of $5 \mathrm{~mm}$ was developed for uterine cavity lesions examination without anesthetics. The effective focus length of the optical imaging system is $1.2 \mathrm{~mm}$, the depth of field $10-100 \mathrm{~mm}$, the maximum field curvature $0.1 \mathrm{~mm}$, and the resolution capability of image system 145 cycles $/ \mathrm{mm}$. CMOS image sensor is with a diagonal dimension of 1/10 in. The illumination system uses the crescent-shaped optical fiber bundle as light transmission and metal halide lamps, high illuminance and high color temperature, as light source. Filter denoise, image enhancement and distortion correction are performed on video image to get a clear, distortionless and high resolution image where blood vessels and tissue morphology of uterine are displayed in real time. The medical electronic endoscope can offer doctors an effective means and reliable bases to early diagnose uterine diseases, especially early stage malignant tumors. Some simple thoracoscopic and laparoscopic operations were simulated to test the reliability and surgeon's acceptability.
\end{abstract}

\section{Introduction}

Endoscopic surgery is a popular approach in many fields of surgery in the present time. Medical electronic endoscope plays an important role in complicated clinical practice. When medical electronic endoscope is inserted into human body doctors can directly observe the tissue morphology and pathological changes of the body cavity and internal organs. Therefore the disease can be highly efficient and accurately diagnosed with endoscope for the early detection of fatal diseases [1, 2]. Minimally invasive surgery with medical electronic endoscope possesses the advantages of little damage, light pain and quick recovery ${ }^{[3,4]}$, its use has been widened to include regular checkups.

The development history of medical endoscope experiences three periods: rigid endoscope, fiber endoscope and electronic endoscope. In 1983, the optical fiber and rigid tube of endoscope image transmission were replaced by the image sensors of charge couple device (CCD) researched and produced by Welchallyn Company of American, which declared the birth of electronic endoscope and realized the historic breakthrough of endoscope. The principle of electronic endoscope is that object is imaged on the photosensitive surface of CCD by the objective lens and CCD converts light signals into electrical ones which are transmitted into the video processor by cable and displayed on the monitor, and then high definition and vivid image is reconstructed from electrical signals by digital image processor (DSP). The image quality directly influences the application of endoscope and also marks the development process of the endoscope technology. Electronic endoscope image is more clear, bright, vivid, and has higher resolution and signal-to-noise ratio than fiber endoscope. By means of electronic endoscope doctors can find the pathological changes undetected with fiber endoscope, which improves detectable rate of diseases, especially of early stage tumor ${ }^{[5-8]}$. One of the most important application fields of electronic endoscope is the early diagnosis of various malignant tumours in womb, including cervical cancer, ovarian cancer, endometrium cancer and so on. Moreover, the suffering of the patients can be relieved because of small outside diameter of electronic endoscope. Hence, electronic endoscope is being applied more and more widely in the clinical diagnosis and treatment medicine ${ }^{[9]}$. 


\section{System}

In order to satisfy the requirements of the diagnosis of uterine cavity lesions without anesthesia, this paper developed a low distortion, high-definition and non-blind medical electronic endoscope with an outside diameter of $5 \mathrm{~mm}$. The overall structure of the electrical endoscope is shown in Fig.1. It mainly consist of four parts, including an front handheld optical image sensor module with three operating key, an illumination system with cold light source, an image processing system for image enhancement, edge sharpening and distortion correction.

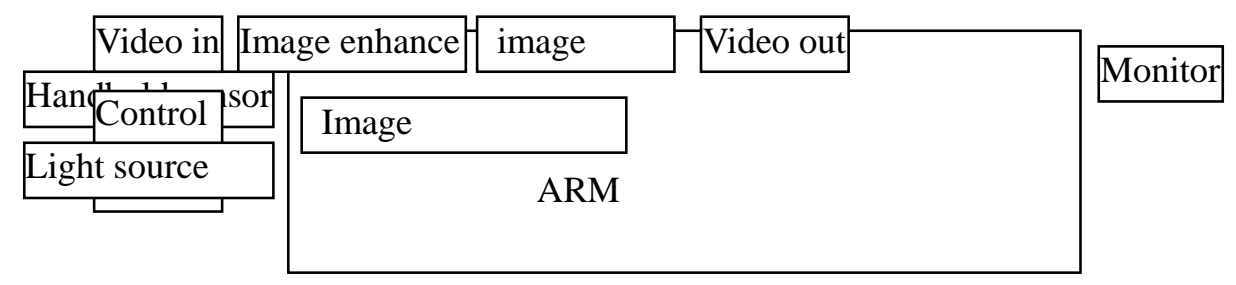

Figure 1. System overall structure schematic drawing of electronic endoscope

The system has introduced the path breaking medical endoscope under new ARM platform with optional High Definition Recording.

Table 1 system specifications

\begin{tabular}{|l|l|l|}
\hline Number & Parameter & Specifications \\
\hline 1 & Optical system & RGB prism method \\
\hline 2 & Resolution & $1920(\mathrm{H}) \times 1080(\mathrm{~V})$ \\
\hline 3 & Lens Mount & C-Mount \\
\hline 4 & Optical format & $35 \mathrm{~mm}\left(36.43 \times 27.62 \mathrm{~mm}^{2}\right)$ \\
\hline 5 & Shutter type & Global shutter \\
\hline 6 & Frame rate & $60 \mathrm{fps}$ \\
\hline 7 & Sensitivity & $20001 \times$ F10/3200 \\
\hline
\end{tabular}

Ergonomic water proof head with 3 buttons. The image sensor is a complementary metal-oxide semiconductor (CMOS) sensor with a resolution of 1920 pixels vertically and 1080 pixels horizontally, coupled with High Sensitivity performance of 2000 1x F10/3200 ${ }^{\circ}$ providing the optimal video quality required for medical endoscope video systems. The refreshing rate is 60 frames per second. The body of the rigid part is made of aluminum material in order to improve heat dissipation.

Video signal processing is done in high-performance digital process of newly developed. Progressive processing improved detail and dynamic resolution. The high-sensitivity performance (2x higher) under low light environments is vastly improved and the reflections are significantly reduced through Digital Matrix Processing.

The quality of visual resolution and depth of field are significantly improved by the special image corrector. A newly developed gradation corrector achieves a higher contrast image without halo or black defects. Also improves the contrast level in the fine details of the picture. Automatic Electronic shutter (Shutter Iris) function maintains the optimized performance in variety of lighting situations. A deep depth of field accurate iris control is possible with Auto-iris camera adaptors or 
Auto-iris lenses.

The center of video image of the screen can be zoomed up to maximum four times by using digital zoom function. The gain can be adjusted automatically to provide the proper brightness. Facility to invert or flip the image allow surgeon to operate from opposite side of camera. The captured images can be flipped horizontally and vertically by the digital processing to correct TV images against the optical inversion caused by optical instruments. It is remote controllable via an external optional unit such as a foot switch or handhold device. Preset parameters for different surgeries help get optimum image in all surgeries. 4 scene files are provided to keep pre-set memories for functions and controls. When correction/function setting is changed, it can also be saved and loaded to USB memory or SD card, easily set back to the original settings. Image Freeze Function is provided for patient education and teaching purpose. Pressing the Freeze button on the front panel or handhold will hold the current image. This allows the detailed checking of situations where the motion could hide the details. The camera head and camera control unit use an antibiotic to help to improve infection control. Optional APP is available to control camera from telephone. The functions of camera can also be controlled through touch screen or keyboard besides entering patient data.

The camera equips with a variety of video signal outputs such as digital $0.8 \mathrm{Vp}-\mathrm{p} / 75 \Omega \mathrm{HD}$ signal (HD-SDI 1920×1080i/p), NTSC/PAL signal (VBS, S-VIDEO), HDMI and Ethernet interface. Monitors provide good color reproduction, higher contrast ratio and faster response time. Monitors have on screen display to change parameters to personalize settings for color, gamma, aspect ratio, etc.

Due to the medical electronic endoscope used in darkened and closed environment inside human body, it is necessary to illuminate the tissues in the body with an external light source when the optical system images them. The performance of the lighting system directly affects the image quality, and then the accuracy of diagnosis. The rise of metal halide lamp and LED provides medical electronic endoscope with more illumination methods which can be chosen according to different needs and local environment. Table 1 lists color temperature, lifetime, volume, power consumption of the three light sources. Although LED light source low power consumption, small size and long service life, its illumination is lower than metal halide lamp. When light source is placed in the front end of electronic endoscope, the heat from the light source will burn body tissue, so the light source have to be installed in the back end and the beams travel to the front end with fiber optic bundle designed various shapes and sizes as needed, which requires a high illuminance light source. Therefore the metal halide lamp, high color temperature and high illuminance, is chosen as the light source, with a color temperature of 5500-6400 K corresponds to sunlight and is available with up to $300 \mathrm{~W}$, with longer lifetime of the lamp compared with halogen.

Table 2 The performance comparisons of various light sources

\begin{tabular}{|l|l|l|l|l|l|}
\hline Light source & Illuminance & $\begin{array}{l}\text { Color } \\
\text { temperature/K }\end{array}$ & Life $/ \mathrm{h}$ & $\begin{array}{l}\text { Power } \\
\text { consumption }\end{array}$ & Volume \\
\hline Halogen lamp & Middle & 3300 & 4000 & Maximum & Large \\
\hline Metal halide & Maximum & 7000 & 12000 & Middle & Large \\
\hline LED & Minimum & 5500 & 30000 & Minimum & Minimum \\
\hline
\end{tabular}




\section{Conclusions}

A low distortion, high-definition and non-blind medical electronic endoscope has be developed, which is with a $5 \mathrm{~mm}$ outer diameter of the rigid part inserted into human and suitable for early diagnosis of malignant tumors in uterus without anesthesia. The medical electronic endoscope with thinner flexible tube can alleviate patients' pain and will have a wide range of applications in clinic. Current study demonstrated the feasibility and possibility of wireless technology in the endoscopic surgery. The wireless ENFE may have a role in endoscopic surgery in the future.

The developed medical electronic endoscope is with an outside diameter of $5 \mathrm{~mm}$ and non-blind spot. Fig. 1 is the images of womb under the medical electronic endoscope. The blood vessels and tissue of the uterus can be observed very distinctly. Angiogenesis and morphological changes of uterus mucosal are key prognostic factors of malignant disease. Doctor can early and accurately diagnose uterine diseases according to the medical endoscope image resemble.

\section{Acknowledgements}

The author thanks Jilin Agricultural University undergraduate science and technology innovation fund in 2017.

\section{References}

[1] C. Siristatidis, Chrelicec, Feasibility of office hysteroscopy through the "see and treat technique" in private practice: a prospective observational study, Arch. Gynecol. Obstet. Vol. 282(2010) No. 1, p.22-24.

[2] J.Metello, A. Relva, E. Milheras, Hysteroscopic diagnostic accuracy in post menopausal bleeding, Acta Med. Port. Vol.21(2008) No.5, p.483.

[3] B. Chance, M. Chen, A.E.T. Chiou, Progress in biomedical optics and imaging optics in health care and biomedical optics: diagnostics and treatment, Proc. SPIE. Vol. 5630(2005) No.II, p.938.

[4] S. Angioni, A. Loddo, F. Milano, Detection of benign intracavitary lesions in postmenopausal women with abnormal uterine bleeding: a prospective comparative study on outpatient hysteroscopy and blind biopsy, J. Minim. Invasive Gynecol. Vol.15(2008) No.1, p. 87-91.

[5] A. Ben Arie, S. Tamir, S. Dubnik, Does hysteroscopy affect prognosis in apparent early-stage endometrial cancer? Int. J. Gynecol. Cancer Vol.18 (2008) No. 4, p.813-819.

[6] E. Cicinelli, M. Marinaccio, B. Barba, Reliability of diagnostic fluid hysteroscopy in the assessment of cervical invasion by endometrial carcinoma: a comparative study with transvaginal sonography and MRI, Gynecol. Oncol. Vol.111(2008) No.1, p.55-61.

[7] N.P. Poly'zos, D. Mauri, S. Tsioras, Intraperitoneal dissemination of endometrial cancer cells after hysteroecopy: a systematic review and meta-analysis, Int. J. Gynecol. Cancer Vol.20(2010) No.2, p.261-267.

[8] G.F. Grimbizis, D. Tsolakidis, T. Mikes, A prospective comparison of transvaginal ultrasound, saline infusion sonohysterography, and diagnostic hysteroscopy in the evaluation of endometrial pathology, Fenil. Steril. Vol.94 (2010) No.7, p.2720-2725.

[9] H.M. Fatemi, J.C. Kasius, A. Timmermans, Prevalence of unsuspected uterine cavity abnormalities diagnosed by office hysteroscopy prior to in vitro fertilization, Hum. Reprod. Vol.25(2010) No.8, p.1959-1965. 